\title{
Hepatocellular carcinoma in the setting of interferon- free treatment for chronic HCV hepatitis - experience of a single center
}

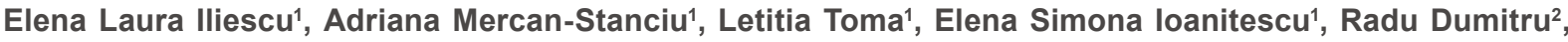 \\ Daniel Rusie ${ }^{3}$ \\ 'Department of Internal Medicine II, Fundeni Clinical Institute, Bucharest 022328, Romania. \\ 2Department of Interventional Radiology, Fundeni Clinical Institute, Bucharest 022328, Romania. \\ ${ }^{3}$ Department of General Surgery, Clinical Emergency Hospital, Bucharest 014461, Romania. \\ Correspondence to: Dr. Elena Laura Iliescu, Department of Internal Medicine II, Fundeni Clinical Institute, Bucharest 022328, \\ Romania. E-mail: laura_ate@yahoo.com

\begin{abstract}
How to cite this article: Iliescu EL, Mercan-Stanciu A, Toma L, loanitescu ES, Dumitru R, Rusie D. Hepatocellular carcinoma in the setting of interferon-free treatment for chronic HCV hepatitis - experience of a single center. Hepatoma Res 2018;4:3. http://dx.doi.org/10.20517/2394-5079.2017.48
\end{abstract}

Received: 7 Nov 2017 First Decision: 26 Dec 2017 Revised: 9 Jan 2018 Accepted: 10 Jan 2018 Published: 18 Jan 2018

Science Editor: Guang-Wen Cao Copy Editor: Jun-Yao Li Production Editor: Cai-Hong Wang

\begin{abstract}
Aim: This study aims to analyze the particularities of hepatitis $\mathrm{C}$ induced hepatocellular carcinoma (HCC), developed during or after treatment with direct-acting antivirals.
\end{abstract}

Methods: We conducted an observational prospective study on 278 patients, who underwent treatment for hepatitis C related liver cirrhosis and respectively for F3 chronic hepatitis $C$. Liver status was assessed using biological parameters and imagistic evaluation (ultrasonography, computed tomography scan, magnetic resonance imaging).

Results: The follow-up time was 14 months. Before therapy, $69.3 \%$ of the cirrhotic patients and $26.7 \%$ of those with F3 degree of liver fibrosis had high levels of alpha-fetoprotein, with no imagistic evidence of HCC. During treatment, HCC was confirmed in 5 patients, 2 of them presenting portal vein thrombosis (PVT). Antiviral therapy was not interrupted. Two patients developed HCC at the end of treatment, while 4 of them were diagnosed with HCC after three months of ending the treatment. Excepting the ones with PVT, all patients underwent trans-arterial chemoembolization.

Conclusion: All patients acquired sustained virological response. The screening for HCC should not be stopped after achievement of sustained virological response. Patients who develop HCC after antiviral treatment often need to be

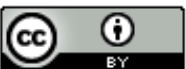

(C) The Author(s) 2018. Open Access This article is licensed under a Creative Commons Attribution 4.0 International License (https://creativecommons.org/licenses/by/4.0/), which permits unrestricted use, sharing, adaptation, distribution and reproduction in any medium or format, for any purpose, even commercially, as long as you give appropriate credit to the original author(s) and the source, provide a link to the Creative Commons license, and indicate if changes were made.

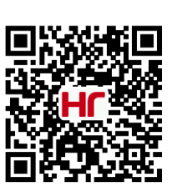


evaluated by magnetic resonance imaging in order to detect the extension of the disease.

Keywords: Hepatitis C virus, direct-acting antiviral therapy, hepatocellular carcinoma, trans-arterial chemoembolization

\section{INTRODUCTION}

Nowadays, hepatitis C virus (HCV) infection represents a global health problem, affecting over 160 million people worldwide ${ }^{[1]}$. The progression of hepatitis $\mathrm{C}$ induced liver disease can be insidious, gradual, during several decades, leading to liver cirrhosis (LC) and hepatocellular carcinoma (HCC). It is estimated that, within 20 years of viral infection, about $20 \%-30 \%$ of subjects will develop LC $^{[2]}$. There is evidence that $2.8 \%$ $11.7 \%$ of the patients with compensated LC will develop hepatic decompensation sooner or later; as for the incidence of HCC, a percentage of $1.8 \%-8.3 \%$ has been reported ${ }^{[3]}$. The use of pegylated interferon (IFN) in combination with ribavirin, in treating the HCV infection, leads to a sustained virological response (SVR) in about $50 \%$ of patients and it is known to have significant side effects ${ }^{[4]}$.

Fortunately, recently there has been a much better understanding of the HCV particularities and structure. The efforts to improve the hepatitis $\mathrm{C}$ management resulted in the discovery and development of directacting antivirals (DAAs), which are meant to interfere with specific steps in HCV replication; in other words, they directly interact with HCV encoded proteins, resulting in the disruption of viral replication ${ }^{[5]}$.

Therefore, DAAs have shown promising effects, increasing the rates of SVR to more than $90 \%$ with notably fewer side effects ${ }^{[6]}$. Although the number of HCV-infected subjects is high, the access to the IFN-free therapy is still limited, due to increased costs and due to the fact that the HCV infection remains highly underdiagnosed ${ }^{[7-9]}$. The use of nucleoside and protease inhibitors has relative contraindications in subjects diagnosed with end-stage renal disease.

It is also worth mentioning that the IFN-free treatments are designed to cure the viral infection, but not the liver disease itself, once the HCV has led to LC or HCC. Furthermore, the risk of complications persists even after achieving SVR, although there is evidence demonstrating improvement in liver function tests after using DAAs ${ }^{[10,11]}$.

Also, even after achieving SVR, re-infection is a possibility that can occur in $10 \%-15 \%$ of patients, especially in individuals at risk, such as intravenous drug users ${ }^{[12]}$.

This study aims to assess the effect of DAAs on liver function and to analyze the particularities of HCC diagnosed during or after treatment with Paritaprevir/Ombistasvir/Ritonavir and Dasabuvir with or without ribavirin (a treatment that is not recommended for patients with decompensated cirrhosis or liver cancer).

\section{METHODS}

In this study, we included a number of 278 patients, all of them infected with HCV genotype $1 \mathrm{~b}$, who received IFN-free treatment with Paritaprevir/Ombitasvir/Ritonavir and Dasabuvir, with or without Ribavirin, for 12 weeks.

An informed written consent was taken from all the participants and all their records were confidential. The scientific purpose of the study, as well as the implications of the therapy itself were presented in detail to each subject, as well as any unexpected research-related risk that may appear.

Viral infection was assessed in each patient by quantitative HCV ribonucleic acid (RNA) tests, describing a high viral load in all patients, with more than $800,000 \mathrm{IU} / \mathrm{L}$. In order to estimate the degree of fibrosis, each 
of the 278 patients underwent a Fibromax evaluation, resulting in 173 patients with F4 degree of fibrosis (cirrhotic patients) and 105 patients with F3 degree of fibrosis. None of the patients underwent liver biopsy for liver evaluation, due to its invasive character and susceptibility of associated complications.

The follow-up time was 14 months (since January 2016 until March 2017). Screening for liver cancer in the subjects included in the study was periodically performed by assessment of alpha-fetoprotein (AFP) levels and abdominal ultrasonography; both parameters were determined before starting the IFN-free therapy, monthly (during therapy), and also every three months afterwards.

According to the protocol of evaluation, the liver assessment was started by performing an AFP determination as well as an abdominal ultrasonography at the beginning of the therapy. If AFP levels were found high (twice the normal value) or ultrasonography abnormalities were spotted, a computed tomography (CT) scan or contrast-enhanced ultrasonography (CEUS) would be performed, in order to obtain more information. CT was not routinely performed as a first-hand screening method for HCC on all patients, at the beginning of the study, due to the potential risk related to ionizing radiation exposure and contrast-induced injury, and also because the use of CT-scan in all the patients was not considered to be cost-effective. Moreover, the AASLD and EASL-EORTC surveillance and diagnostic algorithms in HCC state that only cirrhotic patients are considered candidates for surveillance, and surveillance should be performed with ultrasound every 6 months. It is also worth mentioning that ultrasound examination of all the individuals included in the study was done by highly experienced personnel, with extensive experience in the field of hepatic imaging. Patients with HCC diagnosed in this stage were not included in the study and did not receive any form of IFN-free treatment, as the presence of HCC represents a contraindication for the therapy. AFP and liver enzymes were also determined at week 4 and week 8 of therapy. At the end of treatment (EOT) (week 12), the evaluation protocol included AFP and abdominal ultrasonography. Any abnormality in these parameters would impose a CT scan for further investigation, as well as a magnetic resonance imaging (MRI) (if the CT scan turned out to be inconclusive).

\section{RESULTS}

We prospectively collected and analyzed data from 278 patients infected with HCV genotype 1b, 37.76\% of which (105 individuals) had F3 degree of fibrosis, while 173 of the subjects (meaning approximately 62.24\%) were already in the F4 cirrhosis stage. They were all treated with DAAs (paritaprevir/ombitasvir/ritonavir and dasabuvir, with or without ribavirin) for 12 weeks. Most of the participants (53.24\%) were females; the percent of liver cirrhosis among women was $60.8 \%$. The male population included $46.76 \%$ of the subjects, with a similar percent of $63.8 \%$ cirrhotic participants among the male population. The numeric distribution of patients by gender and degree of fibrosis are shown in Figure 1.

The mean age was $60.29 \pm 11.9$ years. The follow up time was 14 months (from January 2016 to March 2017). Before starting the therapy, $69.3 \%$ of the cirrhotic patients and $26.7 \%$ of the patients with F3 degree of liver fibrosis presented higher than normal levels of AFP, with blood values up to $70 \mathrm{ng} / \mathrm{mL}$. The mean AFP level, at the initiation of therapy, was $13.39 \pm 11.18 \mathrm{ng} / \mathrm{mL}$. Contrast-enhanced ultrasonography was performed for $79 \%$ of patients. Neither CEUS nor the CT scan revealed any HCC nodules at the beginning of therapy. The distribution of patients with high AFP levels, according to gender, fibrosis and means of further evaluation are shown in Figure 2.

During the 4th week of treatment, 5 participants presented elevated levels of AFP. All of them had previously been classified as cirrhotic (F4 on Fibromax evaluation). Abdominal CT scan was therefore performed on all 5 patients, showing single HCC nodules in 2 subjects and multiple HCC nodules in the other three. Furthermore, the CT scan identified signs of portal vein thrombosis (PVT) in 2 of the 5 patients (1 patient 


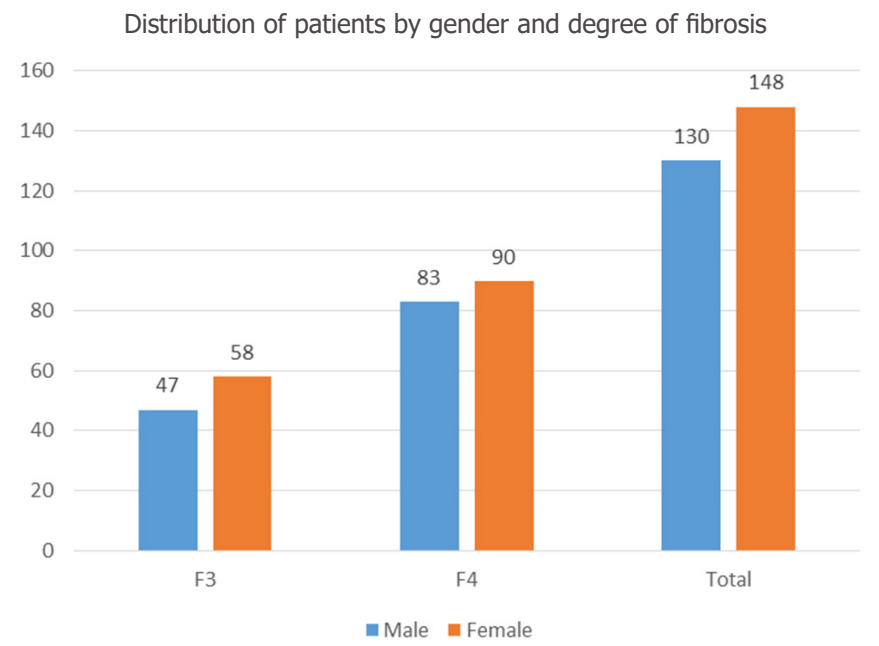

Figure 1. Distribution of patients by gender and degree of fibrosis

Distribution of patients with high AFP levels according to gender, fibrosis, and means of further evaluation

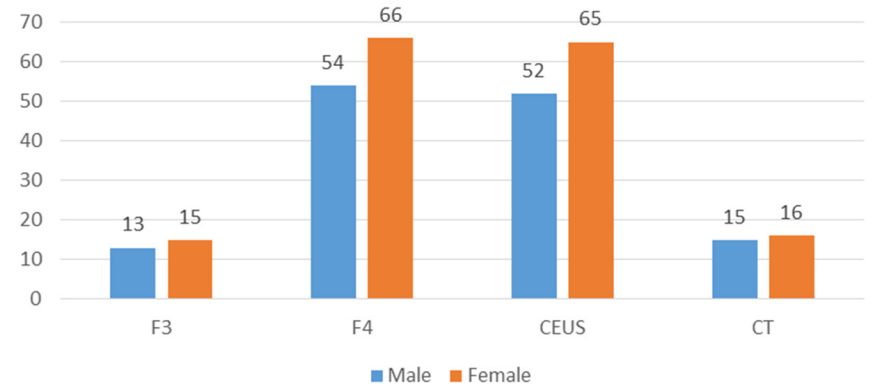

Figure 2. The distribution of patients with high AFP levels, according to gender, fibrosis and means of further evaluation. AFP: alpha-fetoprotein; CT: computed tomography; CEUS: contrast-enhanced ultrasonography

Evolution of patients diagnosed with HCC on treatment

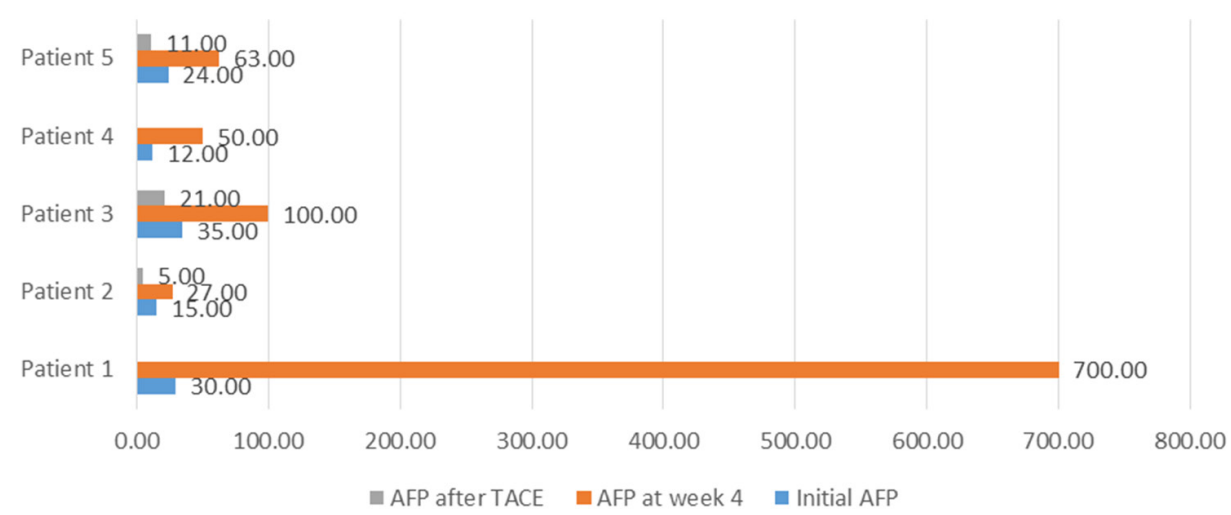

Figure 3. Evolution of patients diagnosed with HCC on treatment. HCC: hepatocellular carcinoma; AFP: alpha-fetoprotein

with single HCC nodule and PVT, and 1 patient with multiple HCC nodules and PVT). The direct antiviral therapy was not interrupted in any of the cases. The 3 subjects diagnosed with HCC and no signs of PVT underwent trans-arterial chemoembolization (TACE) with doxorubicin, while on antiviral therapy, with good outcome. Figure 3 reveals the evolution of the patients who were diagnosed with HCC during treatment. 


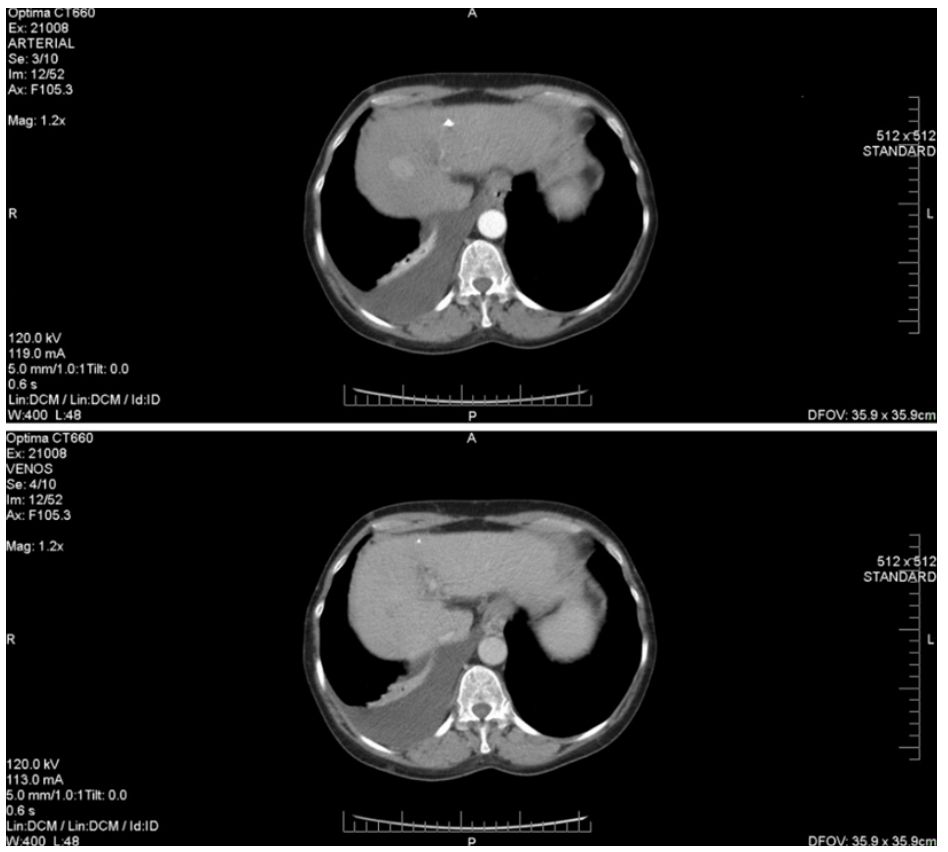

Figure 4. Hepatocellular carcinoma associated with portal vein thrombosis (diagnosed during DAAs treatment). DAAs: direct-acting antivirals

Figure 4 describes the CT image of a HCC diagnosed while the patient was undergoing DAA treatment. We note the presence of a hepatic mass (of about $28 / 23 \mathrm{~mm}$ ), characterized by a typical hyper-enhancement during arterial phase, while, in the portal venous phase, the lesion is rapidly becoming indistinct or hypoattenuating, by comparison to the rest of the liver tissue (venous phase washout). Also, in this patient's case, there are signs of thrombosis of the portal vein trunk, which may be a common phenomenon in individuals with HCC. Compared to HCC without PVT, the association of the two pathological entities is known to be more aggressive and to have a significantly higher chance of complications, as it represents a contraindication for both surgery and TACE. The two patients that were discovered to have HCC and PVC co-occurrence started receiving Sorafenib only after they completed the IFN-free therapy.

At the EOT (week 12), 2 more F4 participants presented higher levels of AFP (twice the values recorded before starting the IFN-free therapy). As they both had previously normal results of the CT scan performed at the beginning of treatment, they were investigated by abdominal MRI, which revealed single HCC nodules, in both cases. These patients refused surgical resection of the nodules and also underwent TACE, with a significant decrease in AFP levels, as it is shown in Figure 5.

At 12 weeks after the EOT, the blood tests performed on the participants revealed increased levels of AFP (up to 10 times the initial value) in other $3 \mathrm{~F} 4$ patients; also, there was one cirrhotic patient who had normal AFP, but in whose case the abdominal ultrasonography showed a single HCC nodule. In the patients with high AFP levels, abdominal CT scan was not able to determine the exact sizes and extension of the nodules, thus an MRI evaluation was required. In all 3 cases, the lesions identified by the MRI were larger and more extensive than the CT scan had anticipated.

One of the three patients diagnosed with HCC at SVR was a 64-year-old female; in her case, the abdominal CT revealed three nodular lesions in segment $8(27 / 30 \mathrm{~mm}, 16 / 18 \mathrm{~mm}$ and respectively $10 / 6 \mathrm{~mm})$, that displayed slight hyper-vascularization in the arterial phase, followed by a late washout, as seen in Figure 6 .

As the patient underwent an MRI investigation, the lesions appeared to be in hypo-signal on T1 and also, it was revealed that the hepatic masses had bigger dimensions than they initially appeared on the CT [Figure 7]. 


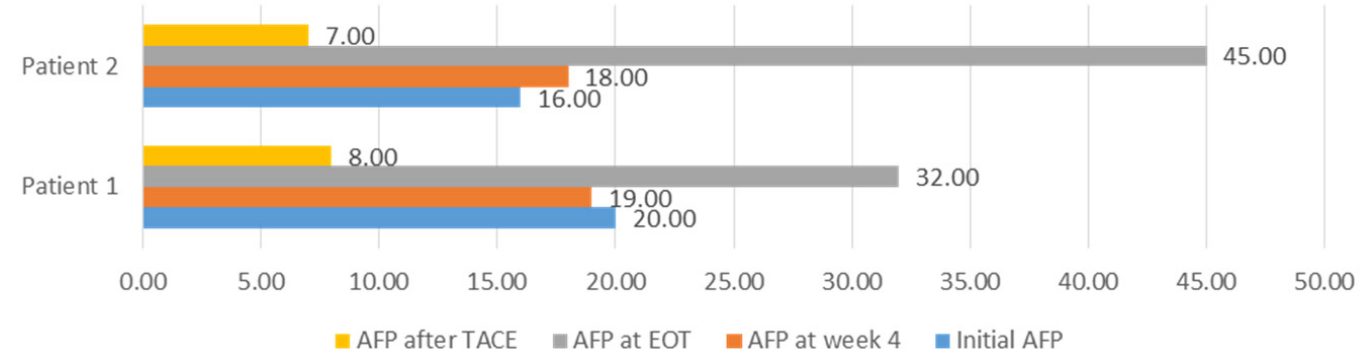

Figure 5. Evolution of patients diagnosed with HCC at the EOT. HCC: hepatocellular carcinoma; AFP: alpha-fetoprotein; TACE: transarterial chemoembolization; EOT: end of treatment

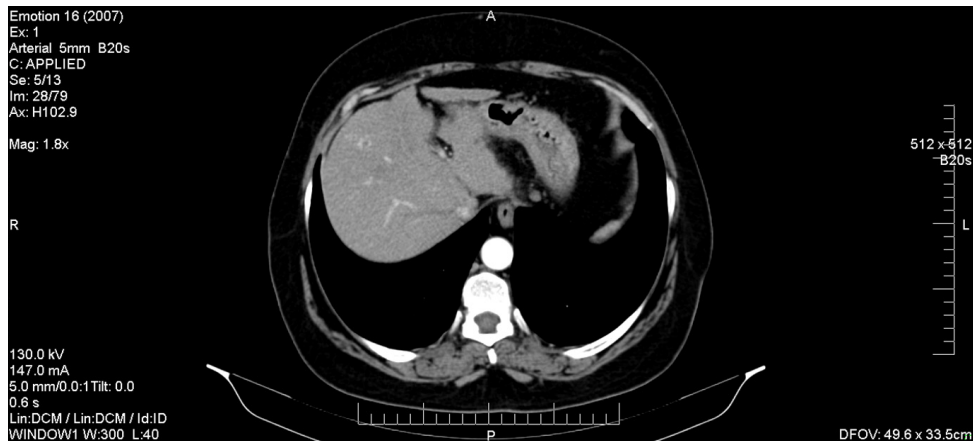

Figure 6. CT image showing three nodular lesions, in the arterial phase. CT: computed tomography

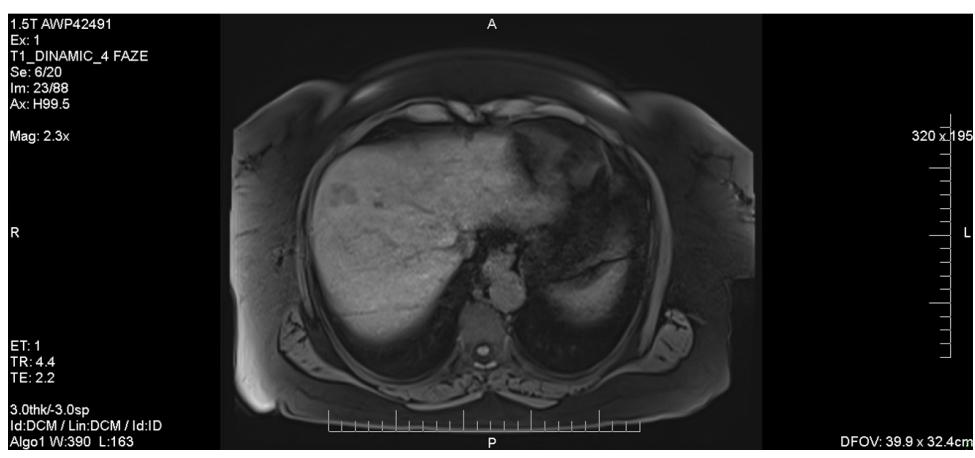

Figure 7. Abdominal MRI showing liver lesions in hypo-signal on $\mathrm{T} 1$ sequences. MRI: magnetic resonance imaging

The MRI exploration also revealed a lesion located in the hepatic dome, also in hypo-signal on T1, which had not been visible on the CT images [Figure 8]; given the presence of the hepatic dome mass, surgical resection was not indicated in this case. This imagistic pattern is consistent with an infiltrative type HCC.

All the patients that presented high levels of AFP at SVR (12 weeks after the EOT), also underwent chemoembolization, with one month follow-up showing no tumor progression and decreased AFP levels. The one patient with normal AFP levels was also evaluated by MRI due to iodine allergy and underwent one session of chemoembolization, with excellent results - no tumor rebound 9 months after the procedure.

Figure 9 shows the evolution of all three patients with elevated levels of AFP at 3 months after ending the antiviral treatment. 


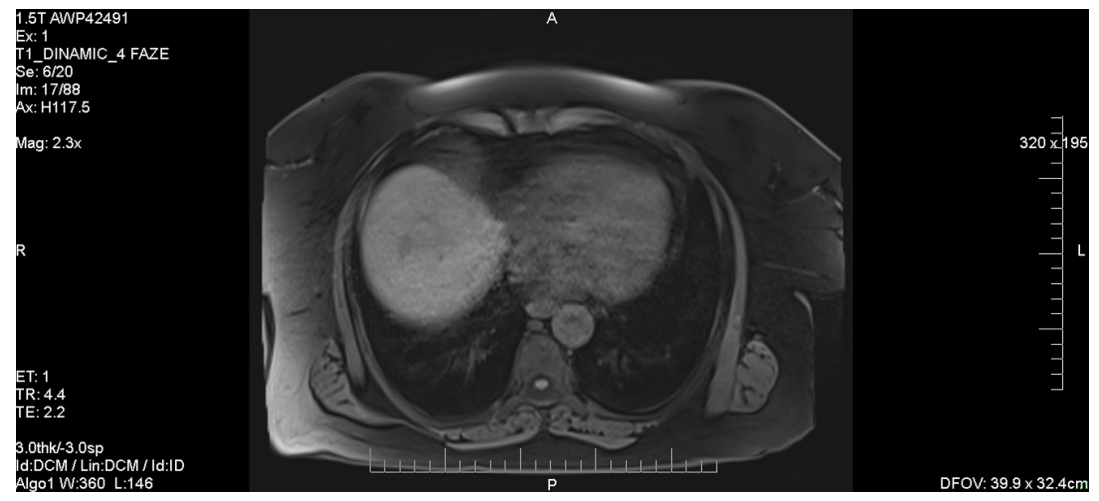

Figure 8. Abdominal MRI showing liver mass in the hepatic dome. MRI: magnetic resonance imaging

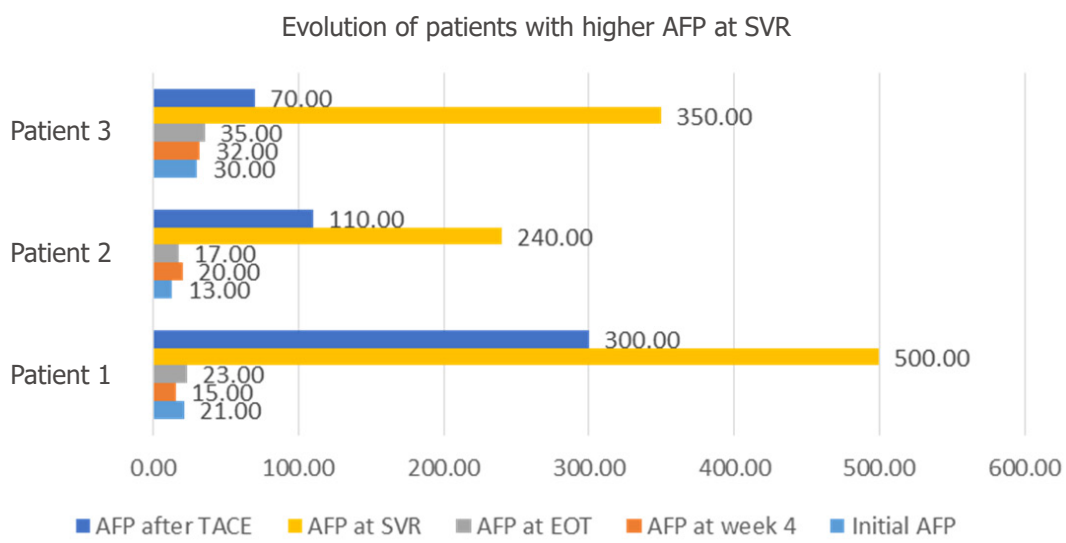

Figure 9. Evolution of patients with higher AFP at SVR. AFP: alpha-fetoprotein; SVR: sustained virological response; TACE: trans-arterial chemoembolization; EOT: end of treatment

Notably, all patients acquired SVR. Overall, the risk of HCC in our study group was $1.51 \%$. When reported to F4 patients, the risk of HCC increases to $6.35 \%$.

\section{DISCUSSION}

It is acknowledged that patients with HCV compensated cirrhosis who achieved SVR due to IFN-based therapy, were shown to be at a lower risk of developing $\mathrm{HCC}^{[13]}$. It was also demonstrated that patients with $\mathrm{HCV}$-induced chronic liver disease had a decrease of the fibrosis level, under DAA treatment ${ }^{[14]}$. For the past several decades, pegylated interferon and ribavirin therapies were used to treat most of the patients with HCV associated liver disease, but they showed various side effects and toxicities.

The use of DAA treatment regimens has opened a new era in the approach of HCV-induced liver disease, reducing the need for liver transplantation ${ }^{[15,16]}$. However, there is evidence of the occurrence or recurrence of HCC in patients with chronic HCV infection, who received DAA therapy, achieving SVR, as shown in a recent publication ${ }^{[17]}$. Therefore, we consider that the association between IFN-free treatment and the development of HCC in patients with chronic HCV infection, should be further investigated and discussed, as it represents a highly important issue in hepatology.

There are several variables associated with an increased risk of developing HCC after SVR, such as advanced liver fibrosis, older age, alcohol abuse, metabolic diseases (especially diabetes mellitus) and the persistence of hepatic inflammation ${ }^{[18,19]}$. A variant in genotype $1 \mathrm{~b} \mathrm{HCV}$ core protein Gln70 (His 70) may also be incriminated in the increase of HCC incidence ${ }^{[20]}$. 
In our study, the occurrence of HCC in patients treated with DAAs was noted during therapy, as well as at the end and also three months after completing it. Older age, comorbidities, advanced fibrosis are all factors that should be taken into consideration when analyzing the increased risk of developing HCC. The mean age of the participants included in our study was $60.29 \pm 11.19$ years, and more than $60 \%$ of them had an F4 degree of fibrosis, which may justify the occurrence of HCC in these cases. The risk of HCC development in patients with compensated cirrhosis in our study group is consistent with literature data ${ }^{[3]}$. However, in spite of the HCC occurrence, SVR was achieved in all the patients that continued antiviral therapy.

It is worth mentioning that, while the alpha interferon based regimens activate natural killer (NK) cells, the DAAs rapidly decrease the levels of HCV RNA, thus leading to a blockage in NK cells activation ${ }^{[21]}$. This way, the protective effect of the inflammatory mechanisms is lost and liver regeneration as well as carcinogenesis may appear ${ }^{[22]}$. There is also evidence showing an elevated level of vascular endothelial growth factor after the initiation of IFN-free treatment ${ }^{[23]}$.

Given the fact that most of the HCC cases found in our study were detected in the first month of therapy, the hypothesis that the tumors were already there, before starting the DAA treatment, simply becoming radiologically detectable after initiating the therapy, should be taken into consideration.

The imaging of HCC is complicated and may have limitations, especially in the early stages, as the tumor has a variety of radiologic appearances and may coexist with regeneration and dysplastic nodules in the cirrhotic liver. In patients with HCC diagnosed during or at the end of DAA therapy, it is most likely that the tumors were already there, mainly due to their dimensions at the time of diagnosis. However, this only emphasizes the importance of ultrasound and AFP follow-up, even during antiviral therapy. In this case, we do not consider that the initial evaluation of these patients should have included a mandatory CT scan, as it has not been proven to be cost-effective; also, a hypothetical mandatory imagistic evaluation would not be CT scan, but liver MRI, with further increases of the imbalance cost-effectiveness. In patients diagnosed with HCC after achieving SVR, it is most likely that the tumors developed during or after antiviral therapy. They were of small dimensions and all presented the infiltrative pattern previously discussed.

Therefore, we recommend the usage of a combination of ultrasound and serum AFP as a primary surveillance method for HCC, especially in cirrhotic patients. If abnormalities are detected by these methods, further exploration by $\mathrm{CT}$ and MRI is required.

In conclusion, the use of DAAs is not associated with a decrease in the development of HCC. Therefore, the screening for HCC should not be stopped after achievement of SVR, as IFN-free treatments cure the viral infection, not the liver disease itself. Patients who develop HCC after antiviral treatment need to be evaluated by MRI in order to detect the extension of the disease as these tumors are more often infiltrative. More studies should be undoubtedly performed, before determining the association between DAA therapy and HCC development.

\section{DECLARATIONS}

\section{Authors' contributions}

Concept and design: Iliescu EL, Mercan-Stanciu A, Toma L

Data acquisition: Mercan-Stanciu A, Toma L, Rusie D

Data analysis: Iliescu EL, Ioanitescu ES, Dumitru R

Manuscript preparation: Iliescu EL, Mercan-Stanciu A, Toma L

Critical revision and finalizing of the manuscript: Iliescu EL 


\section{Data source and availability}

The data obtained through the medical record strictly respected the privacy policy and ethics code of our institute.

\section{Financial support and sponsorship}

None.

\section{Conflicts of interest}

All the authors declare that they do not have anything to disclose with respect to this manuscript.

\section{Patient consent}

An informed written consent was taken from all the participants and all their records were confidential.

\section{Ethics approval}

The study did not require Institutional Review Board approval.

\section{Copyright}

(C) The Author(s) 2018.

\section{REFERENCES}

1. Lavanchy D. Evolving epidemiology of hepatitis C virus. Clin Microbiol Infect 2011;17:107-15.

2. Alberti A, Chemello L, Benvegnù L. Natural history of hepatitis C. J Hepatol 1999;31 Suppl 1:17-24.

3. Alazawi W, Cunningham M, Dearden J, Foster GR. Systematic review: outcome of compensated cirrhosis due to chronic hepatitis C infection. Aliment Pharmacol Ther 2010;32:344-55.

4. Ward RP, Kugelmas M. Using pegylated interferon and ribavirin to treat patients with chronic hepatitis C. Am Fam Physician 2005;72:65562.

5. Scheel TK, Rice CM. Understanding the hepatitis C virus life cycle paves the way for highly effective therapies. Nat Med 2013;19:837-49.

6. Nakamoto S, Kanda T, Shirasawa H, Yokosuka O. Antiviral therapies for chronic hepatitis C virus infection with cirrhosis. World J Hepatol 2015;7:1133-41

7. Edlin BR. Access to treatment for hepatitis C virus infection: time to put patients first. Lancet Infect Dis 2016;16:e196-201.

8. Thomas DL. Global control of hepatitis C: where challenge meets opportunity. Nat Med 2013;19:850-8.

9. Denniston MM, Klevens RM, McQuillan GM, Jiles RB. Awareness of infection, knowledge of hepatitis C, and medical follow-up among individuals testing positive for hepatitis C: National Health and Nutrition Examination Survey 2001-2008. Hepatology 2012;55:1652-61.

10. Conti F, Buonfiglioli F, Scuteri A, Crespi C, Bolondi L, Caraceni P, Foschi FG, Lenzi M, Mazzella G, Verucchi G, Andreone P, Brillanti S. Early occurrence and recurrence of hepatocellular carcinoma in HCV-related cirrhosis treated with direct-acting antivirals. $J$ Hepatol 2016;65:727-33.

11. van der Meer AJ, Berenguer M. Reversion of disease manifestations after HCV eradication. J Hepatol 2016;65:S95-108.

12. Bukh J. The history of hepatitis $\mathrm{C}$ virus $(\mathrm{HCV})$ : Basic research reveals unique features in phylogeny, evolution and the viral life cycle with new perspectives for epidemic control. J Hepatol 2016;65(1 Suppl):S2-21.

13. Conti F, Buonfiglioli F, Scuteri A, Crespi C, Bolondi L, Caraceni P, Foschi FG, Lenzi M, Mazzella G, Verucchi G, Andreone P, Brillanti S. Early occurrence and recurrence of hepatocellular carcinoma in HCV-related cirrhosis treated with direct-acting antivirals. $J$ Hepatol 2016;65:727-33.

14. Cheung MCM, Walker AJ, Hudson BE, Verma S, McLauchlan J, Mutimer DJ, Brown A, Gelson WTH, MacDonald DC, Agarwal K, Foster GR, Irving WL; HCV Research UK. Outcomes after successful direct-acting antiviral therapy for patients with chronic hepatitis C and decompensated cirrhosis. J Hepatol 2016;65:741-7.

15. Yang JD, Aqel BA, Pungpapong S, Gores GJ, Roberts LR, Leise MD. Direct acting antiviral therapy and tumor recurrence after liver transplantation for hepatitis C-associated hepatocellular carcinoma. J Hepatol 2016;65:859-60.

16. Foster GR, Irving WL, Cheung MC, Walker AJ, Hudson BE, Verma S, McLauchlan J, Mutimer DJ, Brown A, Gelson WT, MacDonald DC, Agarwal K; HCV Research, UK. Impact of direct acting antiviral therapy in patients with chronic hepatitis $\mathrm{C}$ and decompensated cirrhosis. J Hepatol 2016;64:1224-31.

17. Reig M, Mariño Z, Perelló C, Iñarrairaegui M, Ribeiro A, Lens S, Díaz A, Vilana R, Darnell A, Varela M, Sangro B, Calleja JL, Forns X, Bruix J. Unexpected high rate of early tumor recurrence in patients with HCV-related HCC undergoing interferon-free therapy. $J$ Hepatol 2016;65:719-26.

18. Balogh J, Victor D 3rd, Asham EH, Burroughs SG, Boktour M, Saharia A, Li X, Ghobrial RM, Monsour HP Jr. Hepatocellular carcinoma: a review. J Hepatocell Carcinoma 2016;3:41-53. 
19. Baumert TF, Jühling F, Ono A, Hoshida Y. Hepatitis C-related hepatocellular carcinoma in the era of new generation antivirals. BMC Med 2017;15:52.

20. Akuta N, Suzuki F, Hirakawa M, Kawamura Y, Sezaki H, Suzuki Y, Hosaka T, Kobayashi M, Saitoh S, Arase Y, Ikeda K, Kumada H. Amino acid substitutions in hepatitis $\mathrm{C}$ virus core region predict hepatocarcinogenesis following eradication of HCV RNA by antiviral therapy. J Med Virol 2011;83:1016-22.

21. Yoon JC, Yang CM, Song Y, Lee JM. Natural killer cells in hepatitis C: current progress. World J Gastroenterol 2016;22:1449-60.

22. Brown RS Jr. The possible association between DAA treatment for HCV infection and HCC recurrence. Gastroenterol Hepatol (N Y) 2016;12:776-9.

23. Villani R, Facciorusso A, Bellanti F, Tamborra R, Piscazzi A, Landriscina M, Vendemiale G, Serviddio G. DAAs rapidly reduce inflammation but increase serum VEGF level: a rationale for tumor risk during anti-HCV treatment. PLoS One 2016;11:e167934. 